\title{
On the packing chromatic number of subcubic outerplanar graphs
}

\author{
Nicolas Gastineau ${ }^{1}$, Přemysl Holub ${ }^{2}$ and Olivier Togni ${ }^{3}$
}

November 8, 2018

\begin{abstract}
Although it has recently been proved that the packing chromatic number is unbounded on the class of subcubic graphs, there exists subclasses in which the packing chromatic number is finite (and small). These subclasses include subcubic trees, base-3 Sierpiski graphs and hexagonal lattices. In this paper we are interested in the packing chromatic number of subcubic outerplanar graphs. We provide asymptotic bounds depending on structural properties of the outerplanar graphs and determine sharper bounds for some classes of subcubic outerplanar graphs.
\end{abstract}

Keywords: packing colouring, packing chromatic number, outerplanar graphs, subcubic graphs.

AMS Subject Classification: 05C12, 05C15, $05 \mathrm{C} 70$

\section{Introduction}

Throughout this paper, we consider undirected simple graphs only, and for definitions and notations not defined here we refer to [2].

Let $G$ be a graph and $c$ a vertex $k$-colouring of $G$, i.e., a mapping $c: V(G) \rightarrow\{1,2, \ldots, k\}$. We say that $c$ is a packing $k$-colouring of $G$ if vertices coloured with the same colour $i$ have pairwise distance greater than $i$. The packing chromatic number of $G$, denoted by $\chi_{\rho}(G)$ is the smallest integer $k$ such that $G$ has a packing $k$-colouring; if there is no such integer $k$ then we set $\chi_{\rho}(G)=\infty$. For a class of graphs $\mathcal{C}$, we say that the packing chromatic number of $\mathcal{C}$ is finite if there exists a positive integer $k$ such that $\chi_{\rho}(G) \leq k$ for every graph $G \in \mathcal{C}$.

The concept of a packing colouring of a graph, introduced by Goddard et al. in [14] under the name broadcast colouring, is inspired by frequency planning in wireless systems, in which it emphasizes the fact that signals can have different powers, providing a model for the frequency assignment problem. The packing chromatic number of lattices has been studied by several authors: for the infinite square lattice $\mathbb{Z}^{2}$, Soukal and Holub in $\left[22\right.$ proved that $\chi_{\rho}\left(\mathbb{Z}^{2}\right) \leq 17$,

\footnotetext{
${ }^{1}$ LAMSADE UMR7243, PSL, Univ. Paris-Dauphine, France; e-mail: nicolas.gastineau@u-bourgogne.fr

${ }^{2}$ Department of Mathematics, University of West Bohemia; European Centre of Excellence NTIS - New Technologies for the Information Society; P.O. Box 314, 30614 Pilsen, Czech Republic; e-mail: holubpre@kma.zcu.cz

${ }^{3}$ Le2I FRE2005, Université Bourgogne Franche-Comté, F-21000 Dijon, France; e-mail: olivier.togni@u-bourgogne.fr
} 
while Ekstein et al. in [8] showed that $12 \leq \chi_{\rho}\left(\mathbb{Z}^{2}\right)$. Recently, Martin et al. in [20] improve the bounds by showing that $13 \leq \chi_{\rho}\left(\mathbb{Z}^{2}\right) \leq 15$. For the infinite hexagonal grid $\mathscr{H}$, Fiala et al. in [11] showed that $\chi_{\rho}(\mathscr{H}) \leq 7$, Korže and Vesel in [18] proved that $\chi_{\rho}(\mathscr{H}) \geq 7$. Finbow and Rall in [12] proved that the infinite triangular grid $\mathscr{T}$ is not packing colourable, i.e., $\chi_{\rho}(\mathscr{T})=\infty$. Moreover, Korže and Vesel in [19] proved that the infinite octagonal lattice has packing chromatic number 7. The packing chromatic number of the Cartesian product of some graphs was investigated in [4, 11, 17]. Also, the packing chromatic number has been studied for further graph classes in [4, 5, 14, 16, 23]. The computational complexity has been also studied: determining whether a graph has packing chromatic number at most 4 is an NP-complete problem [14] and determining whether a tree has packing chromatic number at most $k$ (with a tree and $k$ on input) is also an NP-complete problem [10].

Sloper in 21] showed that the infinite complete ternary tree $T$ has $\chi_{\rho}(T)=\infty$ while any subcubic tree $T$ is packing 7-colourable, hence it is natural to ask if all graphs with maximum degree 3 (often so-called subcubic graphs) have finite packing chromatic number. This question was raised by Goddard et al. [14]. Recently, a second open question has been proposed about the packing chromatic number of $S(G)$, when $G$ is subcubic [7, 13] $(S(G)$ being the graph obtained from $G$ by subdividing each edge once). Recently, Balogh, Kostochka and Liu [1] proved that, for any integer $k$, almost all cubic graphs of order $n$ and of girth at least $2 k+2$ have packing chromatic number greater than $k$, hence answering negatively the question of Goddard et al. Moreover, an explicit construction of an infinite family of subcubic graphs with unbounded packing chromatic number have been found very recently [3]. Some subclasses of subcubic graphs were also under consideration, see e.g. [4, 5].

Outerplanar graphs form a class of structured graphs (containing the class of trees), which are generally easy to colour. Our aim is to find some classes of subcubic outerplanar graphs, which have finite packing chromatic number. We define these classes by giving restrictions on their structure (number of faces of different types), or, equivalently, on their weak dual. Note that, when a graph is not connected, we can colour each component separately satisfying the distance constraints of a packing colouring and the resulting colouring is packing as well. Thus, throughout the rest of this paper, we will consider connected outerplanar graphs only.

The paper is organized as follows. Section 2 presents an upper bound for 2-connected subcubic outerplanar graphs without internal face, i.e., for which the weak dual is a path. Then, in Section 3, we use results from Section 2 in order to determine asymptotic bounds for some larger classes of subcubic outerplanar graphs restricted by the number of (internal) faces. In Section 4, we improve bounds from Section 3 for some specific classes of subcubic outerplanar graphs with a specific structure. Finally, in the last section, we present lower bounds for the packing chromatic number of subcubic outerplanar graphs and give concluding remarks. Table 1) summarizes the main results of this paper.

\subsection{Preliminaries}

Let $G$ be a graph and $A \subset V(G)$. We denote $G-A$ the subgraph of $G$ after deletion of all vertices of $A$ from $G$ and all edges incident to some vertex of $A$ in $G$. We further denote $G[A]$ the 


\begin{tabular}{|c|c|c|}
\hline Condition on the subcubic outerplanar graph $G$ & $\ell$ & Section \\
\hline$G$ is 2-connected with no internal face & 15 & 2 \\
\hline$G$ is 2 -connected with at most $k$ internal faces & $17 \times 6^{3 k}-2$ & 3 \\
\hline$G$ is connected with at most $k^{\prime}$ faces & $9 \times 6^{k^{\prime}}-2$ & 3 \\
\hline$G$ is 2-connected with one internal face & 51 & 4 \\
\hline$G$ is connected with no internal face and with the block graph a path & 305 & 4 \\
\hline
\end{tabular}

Table 1: Classes of subcubic outerplanar graphs and values of $\ell$ for which every relevant graph $G$ satisfies $\chi_{\rho}(G) \leq \ell$.

subgraph of $G$ induced by $A$, or equivalently, $G[A]=G-(V(G) \backslash A)$. Specifically, for $x \in V(G)$, $G-x$ denotes the subgraph of $G$ after deletion of $x$ and all edges incident to $x$ from $G$.

An outerplanar graph $G$ is a planar graph such that there exists a planar drawing of $G$ for which all vertices belong to the outer face. When it is 2 -connected, it can be represented by a boundary cycle $C$ containing all vertices of $G$, with non-crossing chords dividing the interior of $C$ into faces. A face $F$ of $G$ is called an internal face if $F$ contains more than two chords of $G$, and an end face of $G$ if $F$ contains only one chord of $G$; note that all remaining edges of an end face belong to $C$.

The weak dual of $G$, denoted by $\mathcal{T}_{G}$, is the graph with the set of all faces of $G$, except the outer face, as vertex set, and the edge set $E(G)=\left\{F F^{\prime} \mid F\right.$ and $F^{\prime}$ have an edge in common $\}$. We denote by $u_{F}$ the vertex of $\mathcal{T}_{G}$ corresponding to the face $F$ of $G$ and sometimes we identify a face $F$ and the corresponding vertex $u_{F}$ of $\mathcal{T}_{G}$. It is well known that the weak dual of a connected outerplanar graph is a forest and of a 2-connected outerplanar graph is a tree. Note that an end face of an outerplanar graph $G$ corresponds to a leaf of $\mathcal{T}_{G}$ and that an internal face of $G$ corresponds to a vertex of degree at least 3 in $\mathcal{T}_{G}$. Obviously, every end face of a 2-connected outerplanar graph contains at least one vertex of degree 2.

For a graph $G$, the block graph of $G$, denoted by $\mathcal{B}_{G}$, is the graph where vertices of $\mathcal{B}_{G}$ represent all maximal 2-connected subgraphs of $G$ (usually called blocks) and two vertices of $\mathcal{B}_{G}$ are adjacent whenever the corresponding blocks share a cut vertex.

For any $G_{1} \subset G$, let $N\left(G_{1}\right)=\left\{u \in V(G) \mid u v \in E(G)\right.$ for some $\left.v \in V\left(G_{1}\right)\right\}$ be the neighbourhood of $G_{1}$ in $G$. Specifically, if $G_{1}=\{v\}$, let $N(v)$ denote the neighbourhood of $v$ in $G$. For $X, Y \subseteq V(G)$, a shortest $(X, Y)$-path is a shortest path in $G$ between some vertex of $X$ and some vertex of $Y$. If $X$ contains a vertex $u$ only, then we write $u$ instead of $\{u\}$. Let $d_{G}(u, v)$ denote the distance between two vertices $u$ and $v$ in $G$, i.e., the length of a shortest $(u, v)$-path. Analogously, $d_{G}(X, Y)$ denote the distance between $X$ and $Y$, i.e., the length of a shortest $(X, Y)$-path in $G$.

Let $P_{\infty}$ denote the two-way infinite path, i.e., $V\left(P_{\infty}\right)=\mathbb{Z}$ and $E\left(P_{\infty}\right)=\{i i+1 \mid i \in \mathbb{Z}\}$ and let $P_{\infty}^{+}$denote the one-way infinite path, i.e., $V\left(P_{\infty}^{+}\right)=\mathbb{N}$ and $E\left(P_{\infty}^{+}\right)=\{i i+1 \mid i \in \mathbb{N}\}$.

In our proofs we will use the following statement presented by Goddard et al. in [14].

Proposition A [14]. Let $k$ be a positive integer. Then 
i) Every cycle has packing chromatic number at most 4;

ii) There is a packing colouring of $P_{\infty}$ with colours $\{k, k+1, \ldots, 3 k+2\}$;

iii) If $k \geq 34$, then there is a packing colouring of $P_{\infty}$ with colours $\{k, k+1, \ldots, 3 k-1\}$.

\section{2-connected subcubic outerplanar graphs with the weak dual a path}

In the different proofs of this paper, we say that we denote the vertices of a path of order $n$ by $x^{1}, x^{2}, \ldots, x^{n}$ in an ordering starting by $x$ and finishing by $y$, in the case $x^{i}$ and $x^{i+1}$ are adjacent, for $1 \leq i \leq n-1, x^{i}$ and $x$ denote the same vertex and $x^{n}$ and $y$ denote the same vertex.

The following observation will be used in order to construct some useful shortest path in 2-connected subcubic outerplanar graphs. Moreover, it gives a description of 2-connected outerplanar graphs with the weak dual of these graphs.

Observation 1. Let $G$ be a 2-connected outerplanar graph that is not a cycle. Then $G$ contains at least two end faces. Moreover, if $G$ contains no internal face, then $G$ has exactly two end faces.

Proof. Considering the weak dual, $\mathcal{T}_{G}$ is a tree by connectedness of $G$. Since every nontrivial tree has at least two leaves and each leaf of $\mathcal{T}_{G}$ corresponds to some end face of $G$, each 2connected outerplanar graph that is not a cycle contains at least two end faces. In particular, if $G$ has no internal face, then $\mathcal{T}_{G}$ is a path (the converse also holds), implying that $G$ has exactly two end faces.

We begin this section with the following lemma. This lemma will be used in Sections 3 and 4.

Lemma 2. Let $G$ be a 2-connected subcubic outerplanar graph with no internal face. Let $x, y$ be a pair of vertices of degree 2 in $G$ such that $x$ belongs to one of the end faces of $G$ and $y$ to the other one, and let $P$ be a shortest $x, y$-path in $G$. Then there exists a packing colouring of $G$ such that the vertices of $V(G) \backslash V(P)$ are coloured with colours from $\{1,2,3,4\}$.

Proof. Let $C$ denote the boundary cycle of $G$. If there is no chord in $G$, then $G$ is a cycle and, by Proposition $\AA \mathrm{i}), \chi_{\rho}(G) \leq 4$.

Thus we may assume that $C$ contains some chords in $G$. Note that $C-P$ is not necessarily connected, but, since there is no internal face, each component of $C-P$ is an induced path of $G$. Let $D_{i}, i=1, \ldots, k$, denote the components of $C-P$ in an ordering from $x$ to $y$ (i.e., for $i<j, D_{i}$ has a neighbour in $P$ that is closest to $x$ than any neighbour of $D_{j}$ in $P$ ), and let $l_{i}$ denote the length of $D_{i}$. We further denote the vertices of each $D_{i}$ by $x_{i}^{1}, x_{i}^{2}, \ldots, x_{i}^{l_{i}}$ in an 


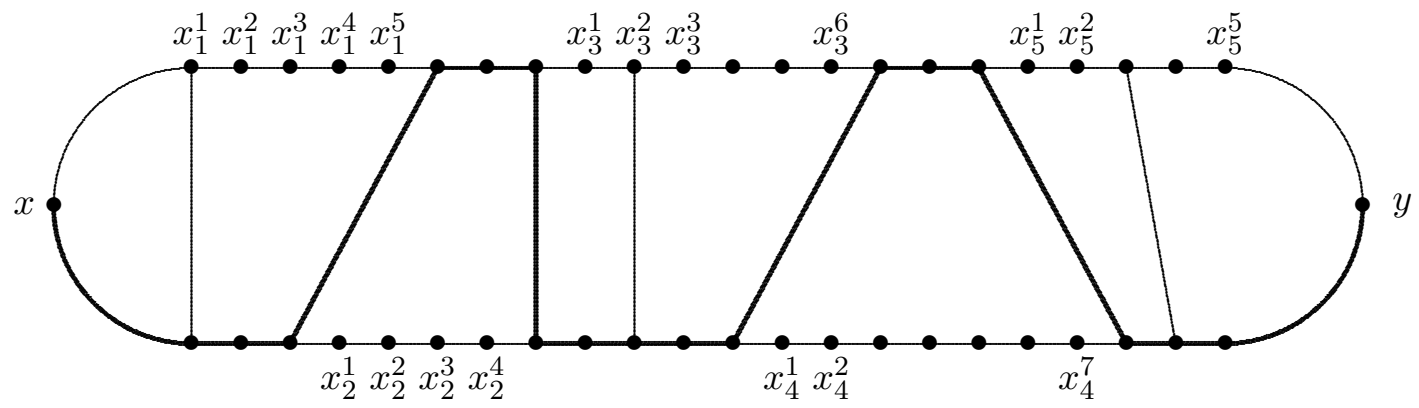

Figure 1: Structure of $G$ in Lemma 2,

ordering starting from a vertex of $D_{i}$ which is closest to $x$ in $D_{i}$. The described structure is shown in Fig. 1, where the thick $x, y$-path depicts $P$.

We colour each component $D_{i}$ of $C-P$ with a pattern $1,2,1,3$ starting from $x_{i}^{1}$ ( $i=$ $1,2, \ldots, k)$, i.e., for each odd $j, x_{i}^{j}$ is coloured with colour 1 , for each $j$ divisible by $4, x_{i}^{j}$ obtains colour 3 , and, for each even $j$ not divisible by 4 , we colour vertex $x_{i}^{j}$ with colour $2, j=1,2, \ldots, l_{i}$. We denote by $\chi$ the defined colouring. Since $P$ is a shortest path and $G$ is subcubic, we are going to show that there is no collision between any pair of vertices coloured with colour 1 or 2 , respectively. Suppose to the contrary that there is a pair of clashing vertices $a$ and $b$ coloured with colour 1. Clearly $a$ and $b$ belong to the same component $D_{i}$ of $C-P$ and $a b \in E(G) \backslash E(C)$ by the definition of $\chi$. But then we get a contradiction with the fact that $D_{i}$ is an induced path of $G$. Now suppose that there is a pair of clashing vertices $a$ and $b$ coloured with colour 2, i.e., $d_{G}(a, b) \leq 2$. Again, $a$ and $b$ must belong to the same component $D_{i}$ of $C-P$, otherwise $d_{G}(a, b)>2$ and we obtain a contradiction. Analogously as for colour 1 we can show that $a b \notin E(G)$. Thus $a$ and $b$ must have a common neighbour $c$ in $G$. From the definition of $\chi$ and also because $D_{i}$ is an induced path in $G, c \notin V\left(D_{i}\right)$, and $c \notin P$ since $G$ is subcubic, a contradiction with the existence of $c$.

Therefore the only possible collision in the defined colouring $\chi$ could be between vertices coloured with colour 3. Analogously as for colours 1 and 2, any pair of clashing vertices $a$ and $b$ cannot be at distance one or two apart. Therefore any such collision happens for $a$ and $b$ with $d_{G}(a, b)=3$. We will check and modify collisions in the defined colouring $\chi$ of the components $D_{1}, D_{2}, \ldots, D_{k}$ of $C-P$ one-by-one starting from $D_{1}$ and from the vertex $x_{i}^{1}$ in each $D_{i}$. Note that, in each step of the modification process, we check the modified colouring, not the original one. The following possible collisions can occur:

Case 1: $a$ and $b$ belong to different components $D_{i}$ and $D_{j}$ of $C-P, i, j \in\{1,2, \ldots, k\}$. Since $d_{G}(a, b)=3$, (up to a symmetry) $a=x_{i}^{l_{i}}, b$ belongs to the chord of $D_{j} \cup P$ which is closest to $a$, and $j=i+1$, otherwise we get $d_{G}(a, b)>3$. Then we can modify the colouring $\chi$ of $D_{j}$ by recolouring vertices $x_{j}^{s}, s=3, \ldots, l_{j}-1$, with $\chi\left(x_{j}^{s}\right):=\chi\left(x_{j}^{s+1}\right)$ and we set $\chi\left(x_{j}^{l_{j}}\right) \in\{1,2,3\}$ depending on the continuation of the pattern $1,2,1,3$ in $D_{j}$.

Case 2: $a$ and $b$ belong to the same component $D_{i}$. Since $d_{G}(a, b)=3, a$ and $b$ must belong to consecutive chords of $P \cup D_{i}$ and there is no vertex between these chords on $P$. We call such a pair of vertices coloured with colour 3 a critical pair. Consider a critical pair $a$ and $b$ such that $a=x_{i}^{m}, b=x_{i}^{n}, m<n$, and that there is no critical pair $a^{\prime}$ and $a$ with 
$a^{\prime}=x_{i}^{o}, o<m$. Then we modify the colouring of the vertices of $D_{i}$ starting at vertex $a$ by

$\underline{3}, 1,4,1, \underline{2}, 1,3,1,2, \ldots$ instead of $\underline{3}, 1,2,1, \underline{3}, 1,2,1,3 \ldots$, i.e., we recolour the vertex $x_{i}^{m+2}$ with colour 4 and we switch colours 2 and 3 of the vertices $x_{i}^{j}$ for even $j>m+2$. Note that the underlined colours represent the critical pair $a$ and $b$. It is easy to verify that vertices coloured with colour 4 are mutually at distance more than 4 apart, implying that there is no collision between any pair of vertices coloured with colour 4 .

After these modifications we obtain a colouring of all the vertices of $G-P$ with colours $\{1,2,3,4\}$ satisfying the distance constraints of a packing colouring.

Theorem 3. If $G$ is a 2-connected subcubic outerplanar graph with no internal face, then $\chi_{\rho}(G) \leq 15$.

Proof. Let $x, y$ be any pair of vertices of degree 2 in $G$ belonging to distinct end faces of $G$. Let $P$ be a shortest $x, y$-path in $G$. By Lemma 2, the vertices of $V(G) \backslash V(P)$ can be coloured with colours from $\{1,2,3,4\}$. Then the colouring can be completed in a packing 15-colouring of $G$ by colouring the vertices along the path $P$ starting at $x$ and using a packing colouring of the infinite path (since $P$ is a shortest path in $G$, then the distance between any pair of vertices of $P$ is the same on $P$ and on $G$ ). For this, we repeat the following pattern with colours from $\{5, \ldots, 15\}$ of length 36 along the vertices of $P$ starting at $x$ :

$5,6,7,9,13,12,5,8,6,10,7,11,5,9,14,6,8,15,5,7,13,10,6,11,5,8,9,7,12,6,5,14,10,15,8,11$

It is easy to check that any two colours $i$ in this repeating sequence are separated by at least $i$ integers.

Note that the previous pattern was found by a computer search.

\section{Asymptotic results for subcubic outerplanar graphs}

The main goal of this paper is to study the finiteness of the packing chromatic number of subcubic outerplanar graphs, i.e, we ask whether the packing chromatic number of an outerplanar graph with maximum degree at most 3 depends on the order of the graph or not. In this section we prove that, for any 2-connected outerplanar graph with a fixed number of internal faces and for any connected outerplanar graph with a fixed number of faces, the packing chromatic number does not depend on the order of the graph.

We begin this section by proving the following useful lemma that will also be used in Section 4. We recall that the weak dual of a 2-connected outerplanar graph is a tree and that $u_{F}$ is the vertex of the weak dual corresponding to the face $F$.

Lemma 4. There exists a packing colouring of $P_{\infty}^{+}$with colours $\{5, \ldots, 15\}$ such that the first vertex along the path is at distance at least $\lceil(i-5) / 2\rceil$ of any vertex of colour $i$. 
Proof. By considering Pattern (1) from the proof of Theorem 3 starting at the first vertex of the path (the vertex of degree 1), we can easily check that the first six vertices of $P_{\infty}^{+}$satisfy the property. Since the colours used in Pattern (1) are bounded by 15, the other vertices (other than the first six vertices) satisfy the property as well.

For positive integers $i, j$ and $k$, let $r_{i, j}^{k} \in \mathbb{Z}$ such that $r_{i, j}^{k} \equiv i-j(\bmod k)$ with minimum absolute value. The value $\left|r_{i, j}^{k}\right|$ corresponds to the distance between two vertices $i$ and $j$ in a cycle $C_{k}$ with vertex set $\{0, \ldots, k-1\}$ (the vertices are enumerated along the cycle). A subset of vertices $A$ of a graph $G$ is a cycle-distance-preserved set if there exists an ordering $v_{A}^{0}, \ldots, v_{A}^{|A|-1}$ of the vertices of $A$ satisfying $d_{G}\left(v_{A}^{j}, v_{A}^{j^{\prime}}\right) \geq\left|r_{j, j^{\prime}}^{|A|}\right|$, for integers $0 \leq j<j^{\prime} \leq|A|-1$.

Lemma 5. For any positive integers $k$ and $n>2$, there exists a packing colouring of the cycle $C_{n}$ with colours from $\{k, \ldots, 6 k+4\}$.

Proof. Let $C_{n}$ be a cycle of length $n$. First, if $n \leq 5 k+5$, then we can colour each vertex of $C_{n}$ with a different colour from $\{k, \ldots, 6 k+4\}$.

Second, if $5 k+5<n \leq 6 k+5$, then we colour $3 k$ consecutive vertices of $C$ with colours $k, \ldots, 3 k-1, k, \ldots, 2 k-1$, and colour the remaining $n-3 k \leq 3 k+5$ vertices of $C_{n}$ with mutually distinct colours from $\{3 k, \ldots, 6 k+4\}$.

Third, suppose $n>6 k+5$. By Proposition A, ii), we can colour $P_{\infty}$ with colours from $\{k, \ldots, 3 k+2\}$. Let $P^{\prime}$ be any subpath of $C_{n}$ on $3 k+2$ vertices. Since the distance between the two ends of $C_{n}-P^{\prime}$ is at least $3 k+3$ in $C_{n}-P^{\prime}$ and exactly $3 k+3$ in $C_{n}$, we can colour the vertices of $C_{n}-P^{\prime}$ with the colours $\{k, \ldots, 3 k+2\}$ (using Proposition A,ii)) and the vertices of $P^{\prime}$ with mutually distinct colours from $\{3 k+3, \ldots, 6 k+4\}$.

A subset of vertices $A$ of a graph $G$ is decomposable into $r$ cycle-distance-preserved sets if there exist $r$ sets of vertices $A_{1}, A_{2}, \ldots, A_{r}$, such that $A_{1} \cup \ldots \cup A_{r}=A$ and for each integer $i, A_{i}$ is a cycle-distance-preserved set. The following lemma will be useful in order to prove Theorems 7 and 8 ,

Lemma 6. Let $G$ be a graph and let $A \subseteq V(G)$ be a subset decomposable into $r$ cycle-distancepreserved sets. The vertices of $A$ can be packing-coloured with colours $\left\{k, \ldots, 6^{r}(k+1)-2\right\}$, for any positive integer $k$.

Proof. We proceed by induction on $r$. For $r=1$, since $A$ is a cycle-distance-preserved set, by Lemma 5, we can colour the vertices of $A$ with colours $\{k, \ldots, 6 k+4\}$. Now suppose that a subset $A \subset V(G)$ is decomposable into $r+1$ cycle-distance-preserved sets. Using induction hypothesis we can colour the vertices of $A_{1}, \ldots, A_{r}$ with colours $\left\{k, \ldots, 6^{r}(k+1)-2\right\}$. For the vertices of $A_{r+1}$, by Lemma 囵, we can use colours $\left\{6^{r}(k+1)-1, \ldots, k^{\prime}\right\}$, where $k^{\prime}=6\left(6^{r}(k+1)-1\right)+4=$ $6^{r+1}(k+1)-2$. Note that we do not need to change colours of the vertices from $\cup_{i=1}^{r}\left(A_{i} \cap A_{r+1}\right)$ (in the case it is not empty). 
The following theorem is one of our main results. It shows that the packing chromatic number of a 2-connected subcubic outerplanar graph with bounded number of internal faces is bounded by a constant.

Theorem 7. If $G$ is a 2-connected subcubic outerplanar graph with $r$ internal faces, then $\chi_{\rho}(G) \leq 17 \times 6^{3 r}-2$.

Proof. Let $F_{1}, \ldots, F_{r}$ denote the $r$ distinct internal faces of $G$, and $u_{F_{1}}, \ldots u_{F_{r}}$ the corresponding vertices of $\mathcal{T}_{G}$ (note that each $u_{F_{i}}$ has degree at least 3 in $\mathcal{T}_{G}$ ). Figure 2 illustrates a subcubic outerplanar graph and its dual. Then, removing the vertices $u_{F_{1}}, \ldots, u_{F_{r}}$ from $\mathcal{T}_{G}$, we obtain a union of disjoint paths. The connected components with one end vertex adjacent in $\mathcal{T}_{G}$ to $u_{F_{i}}$ and the other end vertex adjacent in $\mathcal{T}_{G}$ to $u_{F_{j}}$ are denoted by $U_{i, j}$. In the case $u_{F_{i}}$ and $u_{F_{j}}$ are adjacent, $U_{i, j}$ is not defined (all the vertices in the face "between" $F_{i}$ and $F_{j}$ are colored in Step 3). For any $u_{F_{i}}, i=1, \ldots, r$, the paths with one end vertex of degree 1 and the other one adjacent to $u_{F_{i}}$, are denoted by $U_{i}^{1}, \ldots, U_{i}^{\ell_{i}}$, where $\ell_{i}$ is the number of such paths for $u_{F_{i}}$. Note that some of the paths $U_{i, j}, U_{i}^{q}$ may be trivial or empty.

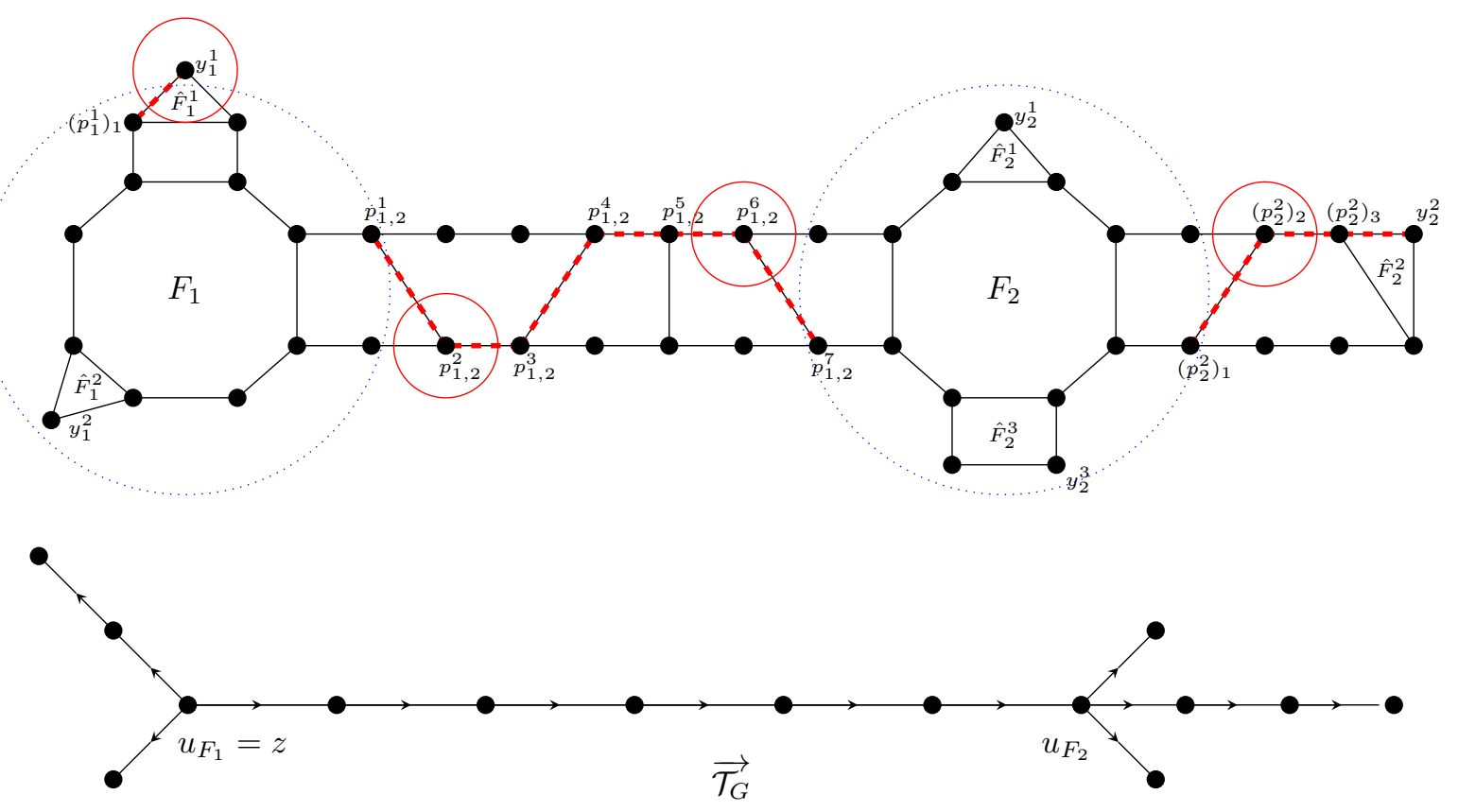

Figure 2: A subcubic outerplanar graph $G$ with two internal faces (on the top) and its oriented dual (on the bottom) (dashed lines : paths $P_{1,2}, P_{1}^{1}$ and $P_{2}^{2}$; vertex in dotted circle : vertex in $B_{1} \cup B_{2}$ or $V\left(F_{1}\right) \cup V\left(F_{2}\right)$; vertex in simple circle: vertex in $\left.D_{1} \cup D_{2}\right)$.

For any $i, 1 \leq i \leq r$, let $B_{i}=N\left(F_{i}\right) \backslash \bigcup_{i=1}^{r} V\left(F_{i}\right)$, and let $B=\bigcup_{i=1}^{r}\left(F_{i} \cup B_{i}\right)$. Let $i$ and $q$ be integers such that $1 \leq i \leq r, 1 \leq q \leq \ell_{i}$. Consider an end face $\hat{F}_{i}^{q}$ in $G$ corresponding to an end vertex of $V\left(U_{i}^{q}\right)$ of degree 1 in $\mathcal{T}_{G}$. We denote by $y_{i}^{q}$ a vertex of $\hat{F}_{i}^{q}$ of degree 2 (note that such a vertex always exists) and by $P_{i}^{q}$ a shortest $\left(B_{i}, y_{i}^{q}\right)$-path in $G$. Let $\left(p_{i}^{q}\right)_{1},\left(p_{i}^{q}\right)_{2}, \ldots$ denote vertices of $P_{i}^{q}$ in an ordering starting from the vertex of $B_{i}$. 
Choose any vertex $z$ of $\mathcal{T}_{G}$ such that $z$ has degree 3 in $\mathcal{T}_{G}$ and let $\overrightarrow{\mathcal{T}_{G}}$ be the digraph obtained from $\mathcal{T}_{G}$ by replacing each edge $u v \in E\left(\mathcal{T}_{G}\right)$ satisfying $d_{\mathcal{T}_{G}}(u, z)<d_{\mathcal{T}_{G}}(v, z)$ with an arc from $u$ to $v$.

Now we consider the paths $U_{i, j}$ in $\mathcal{T}_{G}$. Let $i, j$ be positive integers such that $1 \leq i<$ $j \leq r$ and $U_{i, j}$ is defined and has length at least one. Let $P_{i, j}$ be a shortest $\left(B_{i}, B_{j}\right)$-path and $p_{i, j}^{1}, p_{i, j}^{2} \ldots, p_{i, j}^{l_{i, j}}$ its vertices in an ordering starting from the vertex of $B_{i}$, if $d_{\mathcal{T}_{G}}\left(u_{F_{i}}, z\right)<$ $d_{\mathcal{T}_{G}}\left(u_{F_{j}}, z\right)$, or from the vertex of $B_{j}$ otherwise (by $l_{i, j}$ we mean the order of $P_{i, j}$ ). The upper part of Figure 2 illustrates the notations used in this proof.

Let $P=\left(\bigcup_{1 \leq i \leq r} \bigcup_{1 \leq q \leq \ell_{i}} V\left(P_{i}^{q}\right)\right) \cup\left(\bigcup_{1 \leq i<j \leq k} V\left(P_{i, j}\right)\right)$.

Step 1: Colouring the vertices of $V(G) \backslash(B \cup P)$ with colours $\{1,2,3,4\}$.

We colour the vertices of $V(G) \backslash(B \cup P)$ by colouring each connected component (one by one) of $G-(B \cup P)$ in the same way as in the proof of Lemma 2 , i.e., we use the pattern $1,2,1,3$. Note that the distance between any two vertices from $V(G) \backslash(B \cup P)$ in two different connected components of $G-B$ is at least 5 . Moreover, we proceed as in the proof of Lemma 2 to avoid clashing vertices of colour 3, i.e., we use colour 4 .

Step 2: Colouring vertices of $P$.

Let $i, j, i^{\prime}$ and $q$ be integers such that $U_{i, j}$ and $U_{i^{\prime}}^{q}$ are defined.

For the vertices of $P_{i^{\prime}}^{q}$, we use Pattern (11) and Lemma 4 starting at the vertex $\left(p_{i^{\prime}}^{q}\right)_{3}$. For the vertices of $P_{i, j}$, we use Pattern (11) and Lemma 4, starting at the vertex $p_{i, j}^{3}$ and finishing at the vertex $p_{i, j}^{k_{i, j}-3}$. Note that every vertex of $V\left(\overrightarrow{\mathcal{T}_{G}}\right)$ has in-degree at most one. This property, along with Lemma 4, ensure us that a vertex coloured with colour $a$ in $P_{i, j}, a \in\{5, \ldots, 15\}$, is at distance at least $a+1$ from any other vertex coloured by $a$ in $P_{i, \bar{j}}$, for $1 \leq \bar{i}<\bar{j} \leq r$.

Step 3: Colouring the remaining vertices of $G$.

Let $w_{i, j}$ be a vertex among $\left\{p_{i, j}^{2}, p_{i, j}^{l_{i, j}-2}\right\}$ at distance 2 from a vertex of $V\left(F_{i}\right)$ (when $U_{i, j}$ is defined). Let $D_{i}$ be the set $\left\{\left(p_{i}^{A}\right)_{2} \mid 1 \leq A \leq \ell_{i}\right\} \cup\left\{w_{i, j} \mid U_{i, j}\right.$ is defined, $\left.1 \leq j \leq k\right\}$. Since the sets $V\left(F_{i}\right), B_{i}$ and $D_{i}, 1 \leq i \leq r$, are cycle-distance-preserved sets, the set $\bigcup_{i=1}^{r} V\left(F_{i}\right) \bigcup_{i=1}^{r} B_{i} \bigcup_{i=1}^{r} D_{i}$ is decomposable into $3 r$ cycle-distance-preserved sets. Hence, using Lemma 6 , the remaining uncoloured vertices can be coloured with colours $\{16, \ldots, 17 \times$ $\left.6^{3 r}-2\right\}$.

Sloper in [21] defined an expandable broadcast-colouring of a complete binary tree $T$ as a colouring $c$ of $V(T)$ with colours $1,2, \ldots, 7$ such that:

(i) $\forall u, v \in V(T) c(u)=c(v) \Rightarrow d_{T}(u, v)>c(u)$,

(ii) the root $x$ of $T$ has colour 1 ,

(iii) all vertices at even distance from $x$ have colour 1 ,

(iv) every vertex of colour 1 has at least one child of colour 2 or 3 ,

(v) $c(u)=6, c(v)=7 \Rightarrow d_{T}(u, v) \geq 5$,

(vi) $c(u) \in\{4,5,6,7\} \Rightarrow u$ 's children each have children coloured with 2 and 3 . 
Notice that an expandable broadcast-colouring of a tree is a packing 7-colouring. Sloper has shown that given an expandable colouring of a (complete) binary tree of height $n$, it is possible to create an expandable colouring of a (complete) binary tree of height $(n+1)$ by using the colouring for the tree of height $n$ as a basis [21]. Note that the colouring of a complete binary tree of height 3 that consists in giving the colours 2 and 3 to the two neighbours of $x$, giving the colour 1 to the vertex at distance 2 from $x$, and giving colours from $\{2,3,4,5\}$ to the remaining vertices is an expendable broadcast-colouring. This colouring is described in Figure 3 ,

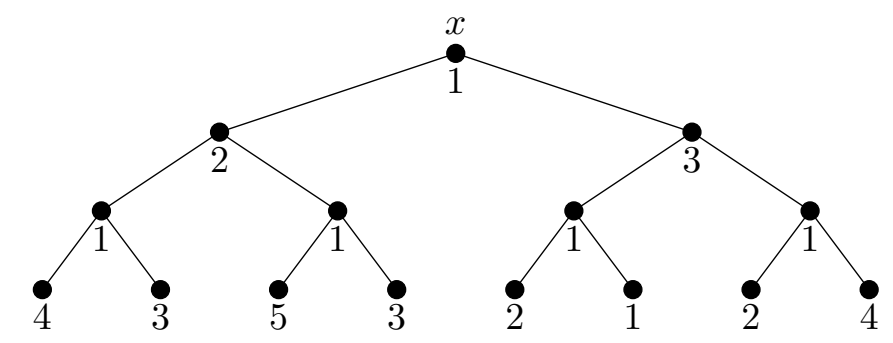

Figure 3: An expendable broadcast-colouring of a complete binary tree of height 3.

The following statement is true for a more general class of graphs than in Theorem 7 since it gives an upper bound for all connected outerplanar graphs (not necessarily 2-connected). However, since the parameter is the number of faces, the bound is weaker than the bound in Theorem 7 .

Theorem 8. If $G$ is a connected subcubic outerplanar graph with $r$ (non external) faces, then $\chi_{\rho}(G) \leq 9 \times 6^{r}-2$.

Proof. Let $F_{1}, \ldots, F_{r}$ denote the $r$ (non external) faces of $G$. The graph $O=G-\bigcup_{i=1}^{r} V\left(F_{i}\right)$ consists of components $O_{1}, O_{2}, \ldots, O_{s}$ such that each $O_{j}(j=1, \ldots, s)$ is a tree. And, since $G$ is subcubic, each $O_{j}$ is subcubic as well. In the weak dual $\mathcal{T}_{G}$ of $G$, choose arbitrary vertex $z$ and let $F_{z}$ denote a face corresponding to $z$ in $G$. We colour the vertices of $G$ in two steps.

Step 1: Colouring the vertices of $O$ with colours $\{1, \ldots, 7\}$.

Consider each component $O_{j}$ of $O$ separately $(i=1, \ldots, s)$ and let $z_{j}$ denote the vertex of $O_{j}$ closest to $F_{z}$. Then we use an expandable broadcast-colouring to colour vertices of $O_{j}$ with colours $1,2, \ldots, 7$ such that the vertices at distance at most 3 from $z_{j}$ are coloured as in Figure 3 (by considering $z_{j}$ as $x$ in this figure). Using the result of Sloper [21, it is possible to extend this packing colouring to the graph $O_{j}$. Note that $z_{j}$ has colour 1 , the neighbour(s) of $z_{j}$ in $O_{j}$ has (have) colour 2 (and 3), vertices of $O_{j}$ at distance 2 from $z_{j}$ are coloured with colour 1 and vertices of $O_{j}$ at distance 3 have colours 2, 3, 4 and 5. Obviously, since $G$ is subcubic, $z_{j}$ is at distance at least 3 from any vertex of any $O_{\ell} \neq O_{i}$. Note that, except one vertex, every vertex of $O$ having a neighbor in $F_{i}$ is at shortest distance of $F_{z}$ (compared to the other vertices in the same component of $O$ ). Consequently, by definition of $z_{j}$, in every face $F_{i}$ there is at most one vertex which has a neighbour in $O$ which is not $z_{j}$ for some $j \in\{1, \ldots, s\}$. 
Let $w_{i}$ be this possible neighbour in $O$. Note that $w_{i}$ can have any colour among $\{1, \ldots, 7\}$. If $w_{i}$ has colour in $\{2,3\}$, then the other vertices of colour 2 or 3 at close distance from vertices of $F_{i}$ are the neighbours of the vertices $z_{j}, j \in\{1, \ldots, s\}$, which are at distance 4 from $w_{i}$. If $w_{i}$ has colour in $\{4,5\}$, then, also, the vertices of colour 4 or 5 at close distance from vertices of $F_{i}$ are the vertices at distance 3 (in $O_{j}$ ) of the vertices $z_{j}, j \in\{1, \ldots, s\}$, and these vertices are at distance 6 from $w_{i}$. Finally, since the vertices at even distance of $z_{j}$ in $O_{j}$ are coloured with colour 1 , the other vertices of colour 6 or 7 are at distance at least 8 from $w_{i}$. Hence, the above defined colouring satisfies the distance constraints of a packing colouring.

Step 2: Colouring the remaining vertices of $G$.

The sets $V\left(F_{1}\right), \ldots, V\left(F_{r}\right)$ are cycle-distance-preserved sets. Hence, by Lemma 6, the remaining uncoloured vertices can be coloured with colours $\left\{8, \ldots, 9 \times 6^{r}-2\right\}$.

\section{Some 2-connected outerplanar graphs with finite packing chro- matic number}

In this section we consider some special classes of subcubic outerplanar graphs for which we can decrease the upper bound on the packing chromatic number given in Theorem 7 .

Theorem 9. If $G$ is a 2-connected subcubic outerplanar graph with exactly one internal face, then $\chi_{\rho}(G) \leq 51$.

Proof. Suppose $G$ is a 2-connected subcubic outerplanar graph with exactly one internal face. Let $C$ denote the boundary cycle of $G$ and $F$ the internal face of $G$. Let $C^{\prime}=\left\{v_{0}, \ldots, v_{N-1}\right\}$ denote the set of vertices which belong to $F$, with $v_{i}$ adjacent to $v_{i+1}$, for $0 \leq i<N$. When $N$ is odd, we suppose that $v_{N-1}$ is a vertex with $d_{G}\left(v_{N-1}\right)=2$. Such a vertex exists since the number of vertices of degree 3 in $C^{\prime}$ is even. By removing the edges of $C \cap F$ from $G$, and by removing the isolated vertices from the resulting graph, we obtain a graph $G^{\prime}$ which is a disjoint union of 2-connected outerplanar graphs having no internal face.

Let $F_{0}, \ldots, F_{s-1}$ denote the 2-connected components of $G^{\prime}$, enumerated in the clockwise order along the cycle $C$ in $G$ (for details, see Fig. 4). Note that, since any $F_{i}$ contains no internal face, each $F_{i}$ has exactly two end faces or $F_{i}$ is a cycle. Let $i$ be an integer with $0 \leq i<s$, and let $u_{i}^{a}$ and $u_{i}^{b}$ denote the two adjacent vertices of degree 3 in $G$ which belong to $V\left(F_{i}\right) \cap C^{\prime}$, as it is depicted in Fig. 4, Let $y_{i}$ be a vertex of degree 2 in the end face of $F_{i}$ which does not contain $u_{i}^{a}$ (for $F_{i}$ a cycle we denote by $y_{i}$ a vertex of $F_{i}$ at maximum distance from $F$ in $G$ ). Let $x_{i} \in\left\{u_{i}^{a}, u_{i}^{b}\right\}$ denote a vertex at minimal distance from $y_{i}$. Finally, let $P_{i}$ be a shortest $\left(x_{i}, y_{i}\right)$-path in $G$. We further denote the vertices of each $P_{i}$ by $x_{i}, p_{i}^{1}, p_{i}^{2}, \ldots, y_{i}$ in an ordering starting from $x_{i}$. Let $D_{i}^{1}, \ldots, D_{i}^{k_{i}}$ denote the connected components of $F_{i}-P_{i}$ with

$D_{i}^{1}$ containing a vertex among $u_{i}^{a}$ and $u_{i}^{b}$ and with $D_{i}^{k}$ being at larger distance than $D_{i}^{k-1}$ from $x_{i}, 2 \leq k \leq k_{i}$. 


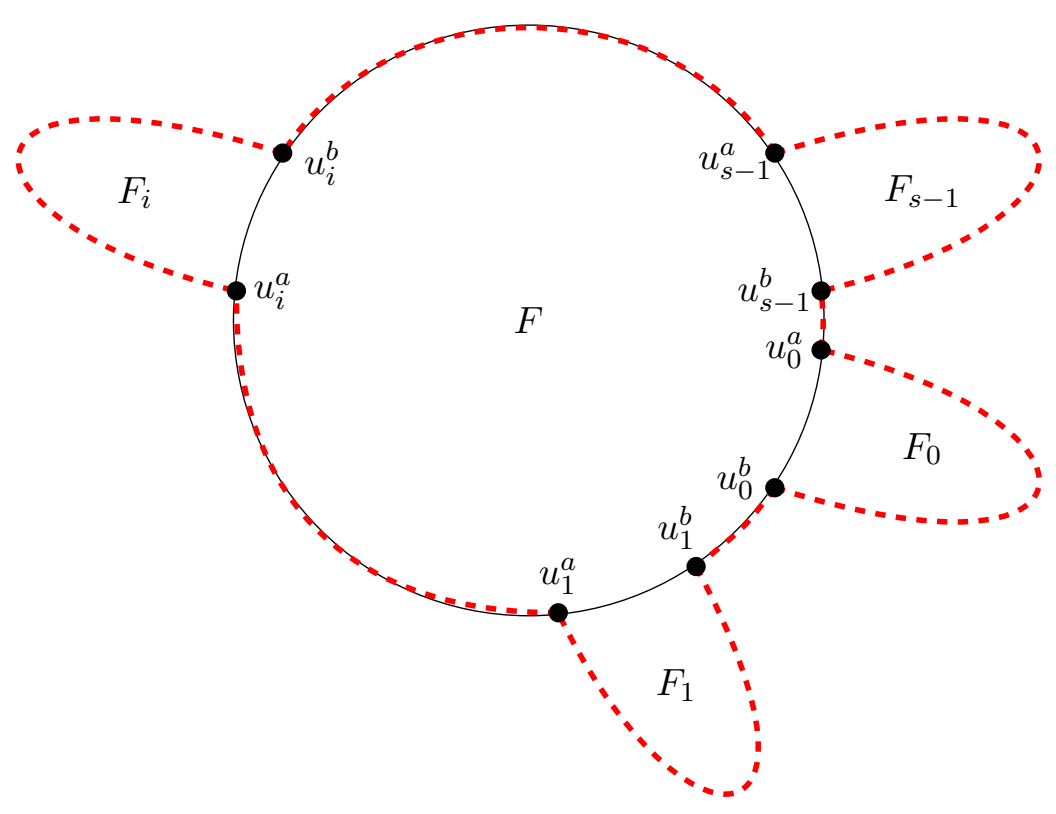

Figure 4: A 2-connected outerplanar graph with one internal face and its different subgraphs ( $C$ is represented by a dashed line).

The proof will be organized as follows. First, we will colour the vertices of $C^{\prime}$. Second, we will colour the vertices of $\cup_{0 \leq i<s} F_{i}-P_{i}$ with colour 1,2 and 3 . Note that the obtained colouring does not necessarily satisfy the distance constraints of a packing colouring of $G$. Third, we will modify colouring of some vertices of $F_{i}-P_{i}(i=0, \ldots, s-1)$ to save colour 1 for some vertices of the paths $P_{i}$ and to prevent collisions in colour 2. Fourth, we will recolour some vertices of $F_{0}, \ldots, F_{s-1}$ with colour 4 in order to satisfy the distance constraints of a packing colouring. Finally, we will colour vertices of the paths $\cup_{0 \leq i<s} P_{i} \backslash\left\{x_{i}\right\}$.

Step 1: Colouring the vertices of $C^{\prime}$ with colours $1,2,29,30, \ldots, 45$.

For integers $j, j^{\prime}$, let $r_{j, j^{\prime}}$ be an integer such that $r_{j, j^{\prime}} \equiv j-j^{\prime}(\bmod N)$ and $-\lfloor N / 2\rfloor \leq$ $r_{j, j^{\prime}} \leq\lfloor N / 2\rfloor$. Note that $d_{G}\left(v_{j}, v_{j^{\prime}}\right)=\left|r_{j, j^{\prime}}\right|$. We begin with a partitioning of $C^{\prime}$ into five subsets: $C_{1}^{\prime}=\left\{v_{j} \mid j \equiv 0(\bmod 2), 0 \leq j<N\right\}, C_{2}^{\prime}=\left\{v_{j} \mid j \equiv 1(\bmod 4), 0 \leq j<N\right\}$, $C_{3}^{\prime}=\left\{v_{j} \mid j \equiv 3(\bmod 12), 0 \leq j<N\right\}, C_{4}^{\prime}=\left\{v_{j} \mid j \equiv 7(\bmod 12), 0 \leq j<N\right\}$ and $C_{5}^{\prime}=\left\{v_{j} \mid j \equiv 11(\bmod 12), 0 \leq j<N\right\}$. Let $m_{k}$ denote the vertex with the largest index in $C_{k}^{\prime}$, for $k \in\{1,2,3,4,5\}$. We use the following patterns to colour the vertices of $C^{\prime}$.

1. if $\left|C_{1}^{\prime}\right| \equiv 0(\bmod 2)\left(\right.$ or $\left.\left|C_{1}^{\prime}\right| \equiv 1(\bmod 2)\right)$, then we colour all vertices of $C_{1}^{\prime}\left(\right.$ or $C_{1}^{\prime} \backslash$ $\left\{m_{1}\right\}$, respectively) with colour 1 ;

2. we colour all vertices of $C_{2}^{\prime} \backslash\left\{m_{2}\right\}$ with colour 2 ;

3. if $\left|C_{3}^{\prime}\right| \equiv 0(\bmod 4)$ (or $\left|C_{3}^{\prime}\right| \equiv 1(\bmod 4)$, respectively), then we use the pattern $29,30,35,36,29,30,35,36, \ldots, 29,30,35,36$ to colour the vertices of $C_{3}^{\prime}$ (or $C_{3}^{\prime} \backslash\left\{m_{3}\right\}$, respectively); 


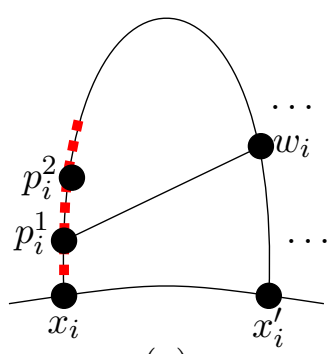

(a)

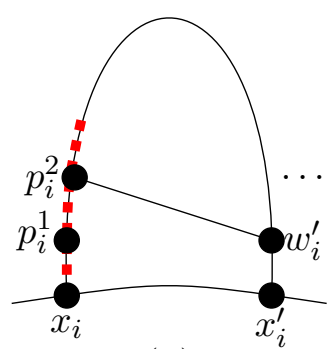

(d)

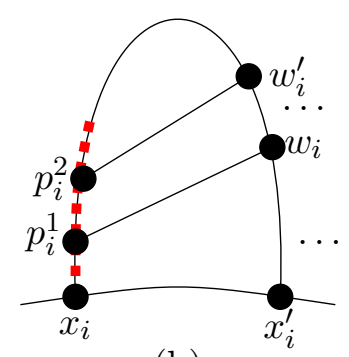

(b)

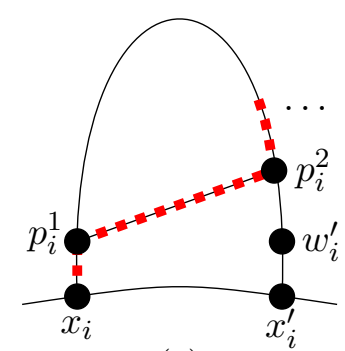

(e)

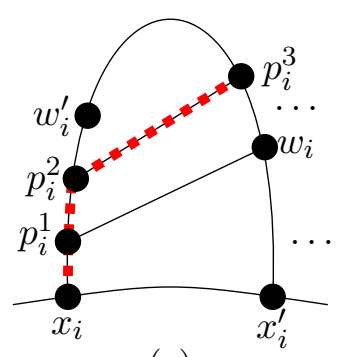

(c)

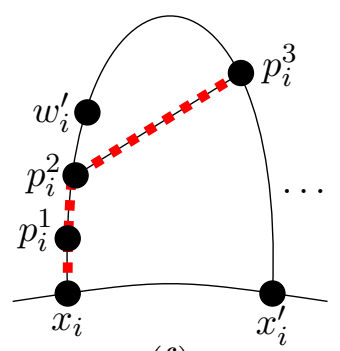

(f)

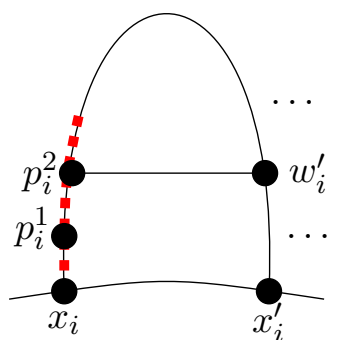

$(\mathrm{g})$

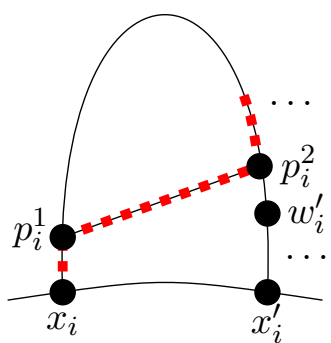

(h)

Figure 5: Eight configurations in step 3 ( $P_{i}$ is represented by a dashed line).

if $\left|C_{3}^{\prime}\right| \equiv 2(\bmod 4)\left(\right.$ or $\left.\left|C_{3}^{\prime}\right| \equiv 3(\bmod 4)\right)$, then we use the pattern $29,30,35,36$, $29,30,35,36, \ldots, 29,30,35,29,30,36$ to colour the vertices of $C_{3}^{\prime}$ (or $C_{3}^{\prime} \backslash\left\{m_{3}\right\}$, respectively);

4. if $\left|C_{4}^{\prime}\right| \equiv 0(\bmod 4)\left(\operatorname{or}\left|C_{4}^{\prime}\right| \equiv 1(\bmod 4)\right)$, then we use the pattern $31,32,37,38$, $31,32,37,38, \ldots, 31,32,37,38$ to colour the vertices of $C_{4}^{\prime}$ (or $C_{4}^{\prime} \backslash\left\{m_{4}\right\}$, respectively); if $\left|C_{4}^{\prime}\right| \equiv 2(\bmod 4)\left(\right.$ or $\left.\left|C_{4}^{\prime}\right| \equiv 3(\bmod 4)\right)$, then we use the pattern $31,32,37,38,31,32$, $37,38, \ldots, 31,32,37,31,32,38$ to colour the vertices of $C_{4}^{\prime}$ (or $C_{4}^{\prime} \backslash\left\{m_{4}\right\}$, respectively);

5. if $\left|C_{5}^{\prime}\right| \equiv 0(\bmod 4)\left(\right.$ or $\left.\left|C_{5}^{\prime}\right| \equiv 1(\bmod 4)\right)$, then we use the pattern $33,34,39,40$, $33,34,39,40, \ldots, 33,34,39,40$ to colour the vertices of $C_{5}^{\prime}$ (or $C_{5}^{\prime} \backslash\left\{m_{5}\right\}$, respectively); if $\left|C_{5}^{\prime}\right| \equiv 2(\bmod 4)\left(\right.$ or $\left.\left|C_{5}^{\prime}\right| \equiv 3(\bmod 4)\right)$, then we use the pattern $33,34,39,40,33,34$, $39,40, \ldots, 33,34,39,33,34,40$ to colour the vertices of $C_{5}^{\prime}$ (or $C_{5}^{\prime} \backslash\left\{m_{5}\right\}$, respectively);

6. when it is necessary, we use the colours $41,42,43,44,45$ to colour the vertices of 


$$
\left\{m_{k} \mid 1 \leq k \leq 5\right\}
$$

One can check that, for any pair of vertices $u, v$ of $C^{\prime}$ with the same colour $k, d_{G}(u, v)>k$. For example, in the pattern 29,30,35,36 of length four, two vertices with the same colour are at distance at least 48 , since the pattern has length four and we colour vertices with the same remainder modulo 12 . The same goes for the pattern $29,30,35,29,30,36$ of length six. Note that, for every pair of vertices $\left(u_{i}^{a}, u_{i}^{b}\right)$, at least one of them is coloured with 1 .

Step 2: Colouring the vertices of $F_{i}-P_{i}$ with colours 1,2 and 3 , for every $i=0, \ldots, s-1$.

Let $x_{i}^{\prime}$ be the vertex among $u_{i}^{a}$ and $u_{i}^{b}$ different from $x_{i}$. Let $l_{i}$ be the order of $D_{i}^{1}$ and let $x_{i}^{\prime}, x_{i}^{1}, \ldots, x_{i}^{l_{i}-1}$ be the vertices of $D_{i}^{1}$ in an ordering starting from $x_{i}^{\prime}$. If $x_{i}^{\prime}$ is coloured with colour 1 , then we use the pattern $3,1,2,1$ to colour the vertices $x_{i}^{1}, \ldots, x_{i}^{l_{i}-1}$. If $x_{i}^{\prime}$ is not coloured with colour 1 , then we use the pattern $1,3,1,2$ to colour the vertices $x_{i}^{1}, \ldots, x_{i}^{l_{i}-1}$. Analogously as in the proof of Lemma 2, we colour vertices of $D_{i}^{j}\left(j=2,3, \ldots, k_{i}\right)$ using the pattern 1,2,1,3 starting from the vertex of $D_{i}^{j}$ at shortest distance from $C^{\prime}$. At this step we do not change the colouring in order to avoid clashing vertices of colour 3 .

Step 3: Recolouring some vertices in $F_{i}$, for every $i=0, \ldots, s-1$.

In this step we deal with possible collisions in colour 2 between vertices of $F$ and vertices of $D_{i}^{1}$ at distance 2 from $F$. We also change colours of neighbours of $p_{i}^{2}$ coloured with 1 since, in Step 5, we will colour $p_{i}^{2}$ with 1 for reducing the number of colours used for the whole graph $G$.

Since we used the patterns $1,3,1,2$ and $3,1,2,1$ to colour the vertices of $V\left(D_{i}^{1}\right) \backslash\left\{x_{i}\right\}$, no vertex at distance 2 from $x_{i}^{\prime}$ has colour 2 . For any $i=0, \ldots, s-1$, let $w_{i}$ denote the vertex of $F_{i}-P_{i}$ at distance 2 from $x_{i}$ and let $w_{i}^{\prime}$ be the possible neighbour of $p_{i}^{2}$ in $F_{i}-P_{i}$.

Case i) $w_{i}$ has colour 2. First suppose that $p_{i}^{2}$ has no neighbour of colour 1 (see Fig. 5(a)) or $p_{i}^{2}$ has a neighbour $w_{i}^{\prime}$ with colour 1 in $D_{i}^{1}$ (see Fig. [5(b)). In both possibilities we recolour the vertices $x_{i}^{j}=w_{i}, x_{i}^{j+1}, x_{i}^{l_{i}-1}$ of $D_{i}^{1}$ with $\underline{4}, 2,1,3,1,2,1 \ldots$ instead of $\underline{2}, 1,3,1,2, \ldots$. Note that the underlined colours belong to the vertex $w_{i}$.

Now we assume that $p_{i}^{2}$ has a neighbour $w_{i}^{\prime}$ of colour 1 which does not belong to $D_{i}^{1}$. Thus $p_{i}^{2} p_{i}^{3}$ is a chord (see Fig. [5(c)). We recolour the vertices $x_{i}^{j}=w_{i}, x_{i}^{j+1}, \ldots, x_{i}^{l_{i}-1}$ of $D_{i}^{1}$ with $\underline{4}, 1,2,1,3, \ldots$ instead of $\underline{2}, 1,3,1,2, \ldots$ (the underlined colours belong to $w_{i}$ ), and the vertices of $D_{i}^{2}$ with pattern $\underline{2}, 1,3,1, \ldots$ instead of $\underline{1}, 2,1,3, \ldots$ (the underlined colours belong to $\left.w_{i}^{\prime}\right)$. Note that if $w_{i}^{\prime}$ had colour 1 , the colour 1 was changed.

Case ii) $w_{i}$ does not have colour 2. If $p_{i}^{2}$ has no neighbour $w_{i}^{\prime}$ of colour 1 , then we do not modify the colouring of $D_{i}^{1}$ in this step. Suppose that $p_{i}^{2}$ has a neighbour $w_{i}^{\prime}$ of colour 1. Suppose that $w_{i}^{\prime}$ is a neighbour of $x_{i}^{\prime}$ (see Fig. $5(\mathrm{~d}, \mathrm{e})$ ). Clearly $x_{i}^{\prime}$ does not have colour 1 and $x_{i}$ has colour 1 (by Step 1 ). Then we modify the path $P_{i}$ by replacing vertex $x_{i}$ with $x_{i}^{\prime}$ and $p_{i}^{1}$ with $w_{i}^{\prime}$ and recolour the modified path $D_{1}^{i}$ with pattern $3,1,2,1,3, \ldots$.

Now suppose that $w_{i}^{\prime}$ is at distance at least 2 from $x_{i}^{\prime}$ and that $p_{i}^{2} w_{i}^{\prime}$ and $p_{i}^{1} p_{i}^{2}$ are not chords (see Fig. $5(\mathrm{f})$ ). We recolour vertices of $D_{2}^{i}$ with pattern $\underline{2}, 1,3,1, \ldots$ instead of $\underline{1}, 2,1,3, \ldots$ If $w_{i}^{\prime}$ is at distance at least 2 from $x_{i}^{\prime}$ and $p_{i}^{2} w_{i}^{\prime}$ is a chord (see Fig. [5(g)), we recolour vertices $x_{i}^{j}=w_{i}^{\prime}, x_{i}^{j+1}, \ldots x_{i}^{l_{i}-1}$ of $D_{1}^{i}$ with $\underline{4}, 1,2,1,3, \ldots$ instead of 
$\underline{1}, 2,1,3, \ldots$ or $\underline{1}, 3,1,2, \ldots$. Finally, if $w_{i}^{\prime}$ is at distance at least 2 from $x_{i}^{\prime}$ and $p_{i}^{1} p_{i}^{2}$ is a chord (see Fig. $5(\mathrm{~h})$ ), then we change the colour of $w_{i}^{\prime}$ to 4 . Note again that, in each possibility, the underlined colours belong to $w_{i}^{\prime}$.

Step 4: Avoid collisions in colouring of vertices of $F_{i}-P_{i}$ for $i=0, \ldots, s-1$.

Now we check and modify (analogously as in the proof of Lemma 2) the defined colouring of $F_{i}-P_{i}$ to avoid collisions between pairs of vertice with the same colour. Obviously, there is no collision between vertices coloured with colour 1 or 2 . Hence the only possible collision is in colour 3. Let $a$ and $b$ be a pair of clashing vertices in colour 3. If $a$ and $b$ belong to the same component $D_{i}^{k}$ of $F_{i}-P_{i}, k, \in\left\{1,2, \ldots, k_{i}\right\}$, then we proceed as in Case 2 of the proof of Lemma 2. Thus we may assume that $a$ and $b$ belong to different components $D_{i}^{k}$ and $D_{i}^{k^{\prime}}$ of $F_{i}-P_{i}, k, k^{\prime} \in\left\{1,2, \ldots, k_{i}\right\}$. If we changed the colouring of $D_{i}^{2}$ in Step 3, then we recolour the vertices of $D_{i}^{2}$ starting from $w_{i}^{\prime}$ (also defined in Step 3) with pattern $2,3,1,2,1,3,1, \ldots$ instead of $2,1,3,1,2,1, \ldots$ Then we proceed as in Case 1 of the proof of Lemma 2 ,

Now we have to check that the vertices coloured with colour 4 in Step 3 are pairwise at distance at least 5, and that the vertices coloured with colour 4 in Step 3 are pairwise at distance at least 5 from the added vertices of colour 4 in Step 4 of applying Lemma 2 , By definition, $w_{i}$ is at distance at most 2 from both $x_{i}$ and $x_{i}^{\prime}$. Moreover, since $w_{i}^{\prime}$ is the neighbour of $p_{i}^{2}$ and since we did not recolour $w_{i}^{\prime}$ with colour 4 in the configurations described in Fig. $5(\mathrm{~d}, \mathrm{e})$ of Step $3, w_{i}^{\prime}$ is either at distance at least 2 from both $x_{i}$ and $x_{i}^{\prime}$ or does not have colour 4 . Thus, the vertices recoloured with colour 4 in Step 3 are at mutual distance at least 5 .

Let $a$ be a vertex of colour 4 from Step 3 (one of $w_{i}, w_{i}^{\prime}$ denoted in Step 3). Suppose that $b$ is a vertex of colour 4 not in $D_{i}^{1}$. The minimal distance between any vertex of $D_{i}^{1}$ and any vertex of $D_{i}^{2}$ is at least 3 . Moreover, because we have proceeded as in the proof of Lemma 2. $b$ is at distance at least 2 from a vertex at minimal distance from $a$. Hence, $d(a, b) \geq 5$.

Now suppose $b$ is a vertex of colour 4 in $D_{i}^{1}$. Since, in every case, $a$ is at distance at least 3 from another vertex of colour 3 in $D_{i}^{1}$, we obtain that $d(a, b) \geq 5$.

Step 5: Colouring the vertices of $P_{i} \backslash\left\{x_{i}\right\}$ with colours 5 to 28 and 46 to 51, for every $i=$ $0, \ldots, s-1$.

We start with colouring of the vertices $p_{i}^{2}$ by 1 for each $i=0, \ldots s-1$. Since we have changed the colours of the eventual neighbours of $p_{i}^{2}$ of colour 1 in Step 3, there are no possible collisions.

For the vertices of $P_{i}$, we use Pattern (10) beginning at the fourth vertex of $P_{i}$, i.e., the vertex $p_{i}^{3}$. By the proof of Lemma 4 , we know that such a colouring satisfies the distance constraints of a packing colouring.

Let $B=\left\{p_{i}^{1} \mid 0 \leq i<s\right\}$. We colour the vertices of $B$ with colours 16 to 28 and (if necessary) 46 to 51 . For integers $j, j^{\prime}$, let $r_{j, j^{\prime}}$ be an integer such that $r_{j, j^{\prime}} \equiv j-j^{\prime}(\bmod s)$ and $-\lfloor s / 2\rfloor \leq r_{j, j^{\prime}} \leq\lfloor s / 2\rfloor$. Note that the vertices $p_{j}^{1}$ and $p_{j^{\prime}}^{1}$ are at distance $2\left|r_{j, j^{\prime}}\right|+1$. We begin by a partitioning of $B$ into three subsets $B_{1}, B_{2}$ and $B_{3}$, with $B_{k}=\left\{p_{i}^{1} \mid i \equiv k-1\right.$ $(\bmod 3), 0 \leq i<s\}, k=1,2,3$. Let $m_{k}\left(m_{k}^{\prime}\right)$ denote the vertex with the largest (second 
largest, respectively) index in $B_{k}$, for $k \in\{1,2,3\}$. We use the following patterns to colour the vertices of $B$.

1. For vertices of $B_{1}$, we use the pattern $16,17,18,16,17,18, \ldots, 16,17,18$. If $\left|B_{1}\right| \equiv$ $1(\bmod 3)\left(\right.$ or $\left.\left|B_{1}\right| \equiv 2(\bmod 3)\right)$, then we erase colours of $m_{1}$ (or of $m_{1}$ and $m_{1}^{\prime}$, respectively).

2. For vertices of $B_{2}$, we use the pattern

$$
19,20,21,25,26,19,20,21,25,26, \ldots, 19,20,21,25,26
$$

when $\left|B_{2}\right| \equiv 0,1,2(\bmod 5)$, or the pattern

$$
19,20,21,25,26,19,20,21,25,26, \ldots, 19,20,21,25,26,19,20,21,25,19,20,21,26
$$

when $\left|B_{2}\right| \equiv 3,4(\bmod 5)$. Then, for $\left|B_{2}\right| \equiv 1,4(\bmod 5)$ we erase colour of $m_{2}$, and for $\left|B_{2}\right| \equiv 2(\bmod 5)$ we erase colours of $m_{2}$ and $m_{2}^{\prime}$.

3. For vertices of $B_{3}$, we use the pattern

$$
22,23,24,27,28,22,23,24,27,28, \ldots, 22,23,24,27,28
$$

when $\left|B_{3}\right| \equiv 0,1,2(\bmod 5)$, or the pattern

$$
27,28,22,23,24,27,28, \ldots, 22,23,24,27,28,22,23,24,27,22,23,24,28
$$

when $\left|B_{3}\right| \equiv 3,4(\bmod 5)$. Then, for $\left|B_{3}\right| \equiv 1,4(\bmod 5)$ we erase colour of $m_{3}$, and for $\left|B_{3}\right| \equiv 2(\bmod 5)$ we erase colours of $m_{3}$ and $m_{3}^{\prime}$.

4. When it is necessary, we use the colours $46,47,48,49,50,51$ to colour the vertices of $\left\{m_{k}, m_{k}^{\prime} \mid 1 \leq k \leq 3\right\}$.

For checking that the defined colouring satisfies the distance constraints of a packing colouring, we recall that any two consecutive vertices in each $B_{k}(k=1,2,3)$ are pairwise at distance at least 7, implying that vertices having the same colour are pairwise at distance at least 19 in $B_{1}$ and at distance at least 31 in $B_{2}$ and in $B_{3}$ (except some vertices of colours from 19 to 24 that can be at distance 25 apart).

Note that in some cases (depending on the size of $B$ and $C^{\prime}$ ) we can decrease the upper bound 51 of Theorem 9. For example, if the internal face $C^{\prime}$ has length $4 k(k \in \mathbb{N})$ and if the number of 2-connected components of $G-C^{\prime}$ is $15 r, r \in \mathbb{N}$, then we can use only 40 colours instead of 51 .

The following statement will be used in the proof of Theorem 11 .

Proposition 10. There is a packing colouring of even vertices of $P_{\infty}$ with colours $\{k, k+$ $1, \ldots, 2 k-1\}$.

Proof. For the colouring of the vertices of the path $P_{\infty}$ we use pattern $1, k, 1, k+1,1, k+$ $2, \ldots, 1,2 k-1$ and after deleting colour 1 we get the required colouring. Note that the distance between any pair of vertices coloured with the same colour in two consecutive copies of this pattern is $2 k$. 
The last class of outerplanar graphs we consider in this paper is a class of not necessarily 2-connected graphs.

Theorem 11. Let $G$ be a connected subcubic outerplanar graph with no internal face such that the block graph of $G$ is a path. Then $\chi_{\rho}(G) \leq 305$.

\section{Proof.}

Let $G$ be a sucubic outerplanar graph, $B_{G}$ the block graph of $G$ and let $B_{1}, \ldots, B_{k}$ denote the blocks of $G$ such that $B_{i}, B_{i+1}$ are consecutive in $B_{G}, i=1, \ldots, k-1$ (i.e., since $\Delta(G) \leq 3$, they are connected by a path which intersects no other block of $G$ ). Let $C_{i}$ denote the boundary cycle of $B_{i}, i=1, \ldots, k$. Since $G$ contains no internal face, each $B_{i}$ contains no internal face as well, implying that every $B_{i}$ which is not a cycle contains exactly two end faces. Let $x_{1}$ denote any vertex of degree two (in $B_{1}$ ) in one end face of $B_{1}, x_{k}$ any vertex of degree two (in $B_{k}$ ) in one end face of $B_{k}$ and let $P$ denote a shortest $x_{1}, x_{k}$-path in $G$. Among all possible choices of the vertices $x_{1}, x_{k}$ we choose $x_{1}^{\prime}$ and $x_{k}^{\prime}$ such that the path $P$ is shortest possible. Let $P_{i}=P \cap B_{i}, i=1, \ldots, k$. Obviously $P$ goes through all the blocks of $G$, hence $P_{i}$ is nonempty for each $i=1, \ldots, k$, every $P_{i}$ is a path since the block graph of $G$ is a path, and each $P_{i}$ is shortest in $G$ since $P$ is shortest in $G$. In an orientation of $P$ from $x_{1}$ to $x_{k}$, we denote by $z_{i}$ the first vertex of $P$ in $B_{i}$ and by $z_{i}^{\prime}$ the neighbour of $z_{i}$ in $B_{i}$ which does not belong to $P$. Note that there must be exactly one such vertex $z_{i}^{\prime}$ in each $B_{i}$ since $P$ is shortest possible and, clearly, each $z_{i}$ must have degree three. For every block which is not a cycle, we further denote by $x_{i}$ any vertex of degree two (in $B_{i}$ ) in one end face of $B_{i}, i=2, \ldots, k-1$ and by $y_{i}$ any vertex of degree two (in $B_{i}$ ) in the end face of $B_{i}$ which does not contain vertex $x_{i}, i=1, \ldots, k$.

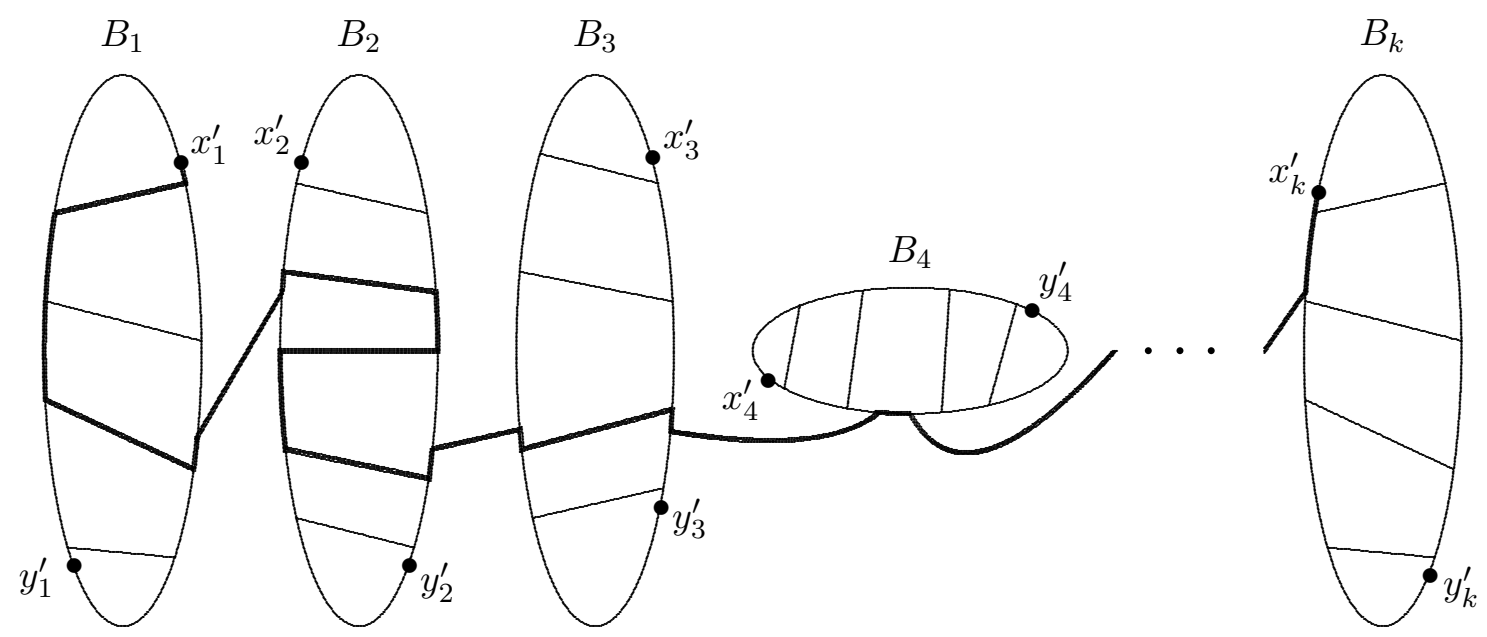

Figure 6: Structure of blocks of the graph $G$ in Theorem 11,

Among all possible choices of the vertices $x_{i}, y_{i}(i=1, \ldots, k)$ we choose $x_{i}^{\prime}, y_{i}^{\prime}$ in such a way that $x_{i}^{\prime}, P$-path and $y_{i}^{\prime}, P$-path, respectively, is shortest possible. For each $i=1, \ldots, k$, let $Q_{2 i-1}$ be a shortest $y_{i}^{\prime}, P$-path in $G$, and let $Q_{2 i}$ be a shortest $x_{i}^{\prime}, P$-path in $G$. Note that $Q_{2}$ and $Q_{2 k}$ are empty and that some other paths $Q_{i}$ may be trivial or empty (e.g., in the case when $B_{i}$ is 


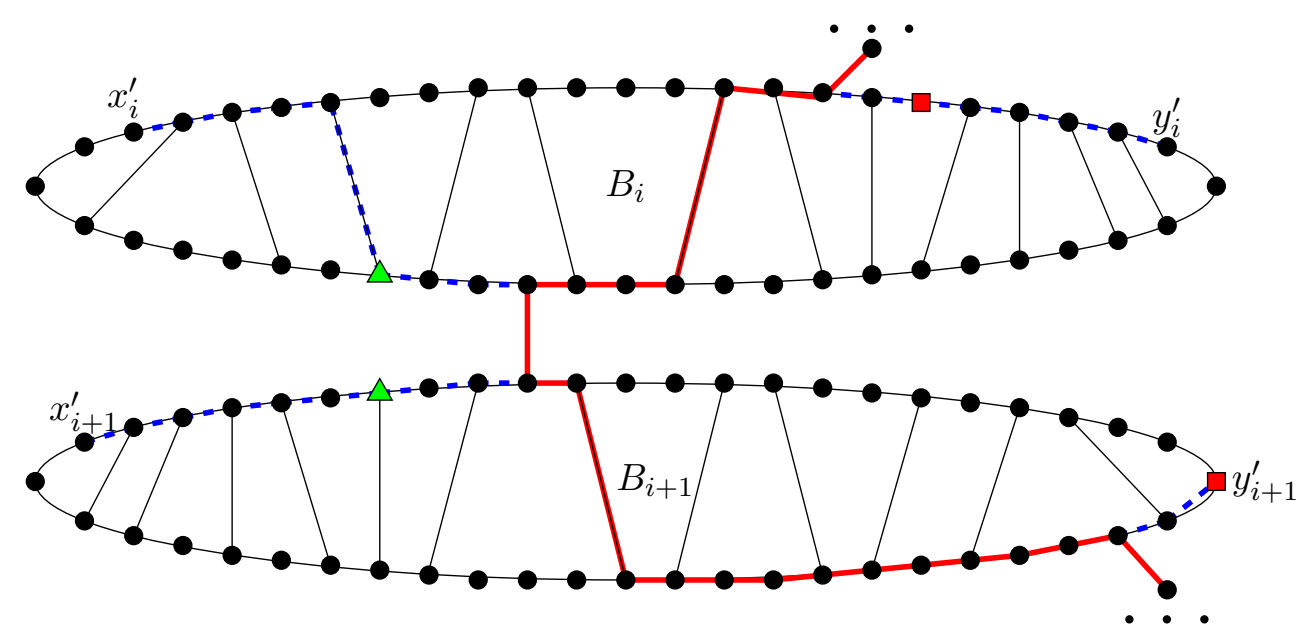

Figure 7: Example of two blocks $B_{i}$ and $B_{i+1}$ and the paths considered in the proof of Theorem 11 in these blocks (thick line: path $P$; dashed lines: paths $Q_{2 i-1}, Q_{2 i}, Q_{2 i+1}$ and $Q_{2 i+2}$ ).

a cycle). The structure of the graph $G$ is depicted in Fig. [6 (the thick path represents the path $P$ ) and the paths $Q_{2 i-1}, Q_{2 i}$ are illustrated in Fig. 7 .

Consider each block $B_{i}$ separately. Note that, for each $i=1, \ldots, k$, the graph $G_{i}=G\left[B_{i}-\right.$ $\left.\left(V\left(P_{i}\right) \cup V\left(Q_{2 i-1}\right) \cup V\left(Q_{2 i}\right)\right)\right]$ consists of path components. Thus, analogously as in the proof of Lemma 2, we can colour vertices of $G_{i}$ with colours $1,2,3,4$ using the periodic pattern 1,2,1,3 and modifications introduced in the proof of Lemma 2, starting at the end face of $B_{i}$ containing vertex $x_{i}$. Note that, if $B_{i}$ is a cycle, $\chi_{\rho}\left(B_{i}\right) \leq 4$. Moreover, we can colour the vertices of $G_{i}$ in such a way that the vertex $z_{i}^{\prime}$ gets colour 1 (if not so, then we can interchange roles of $x_{i}$ and $y_{i}$ for colouring of $V\left(G_{i}\right)$, i.e., we start such a colouring from $y_{i}$ instead of $x_{i}$ ).

Now we check that the defined colouring meets the conditions of a packing colouring. First, there is no collision in colours 1 and 2 , since $\operatorname{dist}_{G}(a, b) \geq 3$ for any $a \in V\left(G_{i}\right)$ and $b \in V\left(G_{j}\right), i<$ $j$. From the modifications described in the proof of Lemma 2, it follows that no end vertex of any path component of any $G_{i}$ is coloured with colour 4 since colour 4 was used for a vertex between vertices of a critical pair belonging to one path component. This implies that the distance between two vertices coloured with 4 which belong to different block $B_{i}, B_{j}$ is at least 5 , hence there is no collision in colour 4 . For colour 3 , since no $z_{i}^{\prime}$ is coloured with colour 3 , there is no collision in colour 3 as well. And since there is no edge in $G$ connecting the path components of the blocks $B_{i}$, the defined colouring meets the distance constraints of a packing colouring of $\bigcup_{i=1}^{k}\left[B_{i}-\left(V\left(P_{i}\right) \cup V\left(Q_{2 i-1}\right) \cup V\left(Q_{2 i}\right)\right)\right]$.

Now we colour the paths $Q_{j}, j=1,2, \ldots, 2 k$. The distance from $P$ to any vertex of $Q_{j}$ in $G$ is the same as on $Q_{j}$, hence each $Q_{j}$ is a shortest path in $G$ between $P$ and the relevant vertex $x_{i}^{\prime}$ or $y_{i}^{\prime}$, respectively. Thus, by Lemma 4 , we can colour the vertices of each path $Q_{2 i-1}$ $(i=1,2, \ldots, k)$ with Pattern (11), starting at the vertex of $Q_{2 i-1}$ at distance two from $P$ (square vertices in Fig. 7). Analogously we can colour the vertices of the paths $Q_{2 i}(i=1,2, \ldots, k)$ using Pattern (1), starting at the vertex of $Q_{2 i}$ at distance three from $P$ (triangle vertices in 


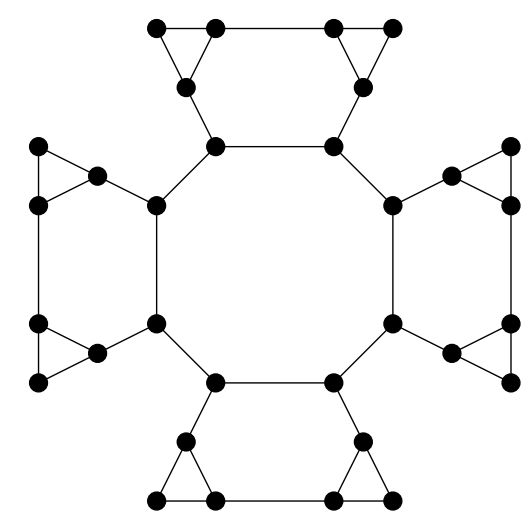

Figure 8: An outerplanar subcubic graph with packing chromatic number 7.

Fig. (7). Then the distance between vertices on distinct paths $Q_{m}, Q_{n}$ coloured with colour 5 is at least $3+1+2$, the distance between vertices on distinct paths $Q_{m}, Q_{n}$ coloured with colour 6 is at least $4+1+3$, etc. Therefore the defined colouring of the paths $Q_{j}, j=1, \ldots, 2 k$ satisfies the distance constraints of a packing colouring.

Now we colour the remaining vertices of $G$. We start with colouring of the path $P$ with a pattern using colours $16,17, \ldots, 50$ by Proposition $\mathrm{A}$ ii). Then we colour all uncoloured vertices of $Q_{j}(j=1, \ldots, 2 k)$ at distance one from $P$ with colours $51,52, \ldots, 152$ by Proposition @iii). For the remaining vertices of $Q_{2 i}$ at distance two from $P$, the distance between any such vertices on $Q_{2 m}$ and $Q_{2 n}(m, n \in\{1,2, \ldots, k-1\}, m \neq n)$ is at least $2|m-n|+4$. Hence we can colour these vertices with colours $153, \ldots, 305$ by Proposition 10 ,

\section{Concluding remarks}

In the previous sections, we have determined some classes of outerplanar graphs with finite packing chromatic number. As for lower bounds, we are (only) able to state the following, where symbols $\square$ and $\otimes$ denote the Cartesian and strong product of graph, respectively (see [15]).

Proposition 12. There exists an infinite family of 2-connected subcubic outerplanar graphs without internal faces and with packing chromatic number 5.

Proof. It has been proven in [14] that $\chi_{\rho}(G)=5$ for $G=P_{n} \square P_{2}$ and $n \geq 6$.

Remark 1 The graph $G$ illustrated in Figure 8 is a 2-connected subcubic outerplanar graph with packing chromatic number 7 . We verified by computer that every proper colouring of $G$ with 6 colours is not a packing colouring and we found a packing colouring of $G$ with 7 colours.

Brešar et al. [4] have proven that for any finite graph $G$, the graph $G \otimes P_{\infty}$ has finite packing chromatic number. The degree of $G \otimes P_{\infty}$ can be arbitrary large. This property illustrates the fact that the degree of a graph is not the only parameter to consider in order to have finite 
packing chromatic number. Maybe the fact that the weak dual is a path (and is not any tree) helps to bound the packing chromatic number. It remains an open question to determine if the packing chromatic number of subcubic outerplanar graphs is finite or not.

\section{Acknowledgments}

The authors thank the referees for their judicious comments and Mahmoud Omidvar for his precious help, in particular for providing Pattern (11) of proof of Theorem 3 . First author was partly supported by the Burgundy Council under grant \#CRB2011-9201AAO048S05587. Second author was partly supported by project P202/12/G061 of the Czech Science Foundation.

\section{References}

[1] J. Balogh, A. Kostochka, and X. Liu: Packing chromatic number of cubic graphs. Discrete Appl. Math. 341(2):474-483, 2018.

[2] J. A. Bondy and U. S. R. Murty: Graph Theory. Springer, 2008.

[3] B. Brešar, J. Ferme: An infinite family of subcubic graphs with unbounded packing chromatic number. Discrete Math. 341:2337-2342, 2018.

[4] B. Brešar, S. Klavžar, and D. F. Rall: On the packing chromatic number of Cartesian products, hexagonal lattice, and trees. Discrete Appl. Math. 155(17):2303-2311, 2007.

[5] B. Brešar, S. Klavžar, and D. F. Rall: Packing Chromatic Number of Base-3 Sierpiski Graphs. Graphs and Combin. 32(4):1315-1327, 2016.

[6] B. Brešar, S. Klavžar, D. F. Rall, and K. Wash: Packing chromatic number under local changes in a graph. Discrete Math. 340:1110-1115, 2017.

[7] B. Brešar, S. Klavžar, D. F. Rall, and K. Wash: Packing chromatic number, (1,1,2,2)colorings, and characterizing the Petersen graph. Aequat. Math. 91:169-184, 2017.

[8] J. Ekstein, J. Fiala, P. Holub, and B. Lidický: The packing chromatic number of the square lattice is at least 12. arXiv:1003.2291v1, 2010.

[9] J. Fiala and P. A. Golovach: Complexity of the packing coloring problem for trees. Discrete Appl. Math. 158:771-778, 2010.

[10] J. Fiala and P. A. Golovach: Complexity of the packing coloring problem for trees. Discrete Appl. Math., 158(7):771-778, 2010.

[11] J. Fiala, S. Klavžar, and B. Lidický: The packing chromatic number of infinite product graphs. European J. Combin. 30(5):1101-1113, 2009.

[12] A.S. Finbow and D.F. Rall: On the packing chromatic number of some lattices. Discrete Appl. Math. 158:1224-1228, 2010. 
[13] N. Gastineau and O. Togni: S-Packing Colorings of Cubic Graphs. Discrete Math. 339:2461-2470, 2016.

[14] W. Goddard, S. M. Hedetniemi, S. T. Hedetniemi, J. M. Harris, and D. F. Rall: Broadcast chromatic numbers of graphs. Ars Comb. 86:33-49, 2008.

[15] R. Hammack, W. Himrich and S. Klavžar, Handbook of Graph Product, 2nd edition, CRC Press, 2011.

[16] J. Ekstein, P. Holub, and O. Togni: The packing colouring of distance graph $D(k, t)$. Discrete Appl. Math. 167:100-106, 2014.

[17] Y. Jacobs, E. Jonck, and E. Joubert: A lower bound for the packing chromatic number of the Cartesin product of cycles. Centr. Eur. J. Math. 11(7):1344-1357, 2013.

[18] D. Korže and A. Vesel: On the packing chromatic number of square and hexagonal lattice. Ars Mathematica Contemporanea 7:13-22, 2014.

[19] D. Korže and A. Vesel: $(d, n)$-packing colorings of infinite lattices. Discrete Appl. Math. 237:97-108, 2018.

[20] B. Martin, F. Raimondi, T. Chen, and J. Martin: The packing chromatic number of the infinite square lattice is between 13 and 15. Discrete Appl. Math. 225: 136-142, 2017.

[21] C. Sloper: Broadcast-coloring of trees. Reports in Informatics 233:1-11, 2002.

[22] R. Soukal and P. Holub: A Note on Packing Chromatic Number of the Square Lattice. Electron. J. of Combinatorics 17:447-468, 2010.

[23] O. Togni: On packing Colorings of Distance Graphs. Discrete Appl. Math. 167:280-289, 2014. 